\title{
Are Neutrophil-to-Lymphocyte and Platelet-to-Lymphocyte Ratios Associated with Mortality in Pediatric Trauma Patients? A Retrospective Study
}

\author{
Yusuf Kenan Tekin* \\ Department of Emergency, Sivas Cumhuriyet University, Imaret/Merkez/Sivas, Turkey
}

\begin{abstract}
Background: There are very limited data on the prognostic capacity of the neutrophil-to-lymphocyte ratio (NLR) and platelet-to-lymphocyte ratio (PLR) for the systemic inflammatory response in pediatric trauma (PT) patients. The purpose of this study was to evaluate the prognostic ability of NLR and PLR on mortality in pediatric trauma patients.

Methods: This study looked at 358 PT patients who were admitted to the Cumhuriyet University Hospital's Emergency Department between January 2010 and June 2018. The NLR and PLR were calculated by dividing the blood neutrophil count and blood platelet count, respectively, by the lymphocyte count, at the time of admission. After performing a stepwise logistic regression analysis to determine the predictive factors on the mortality risk of post-traumatic systemic inflammatory response syndrome (SIRS), receiver
\end{abstract}

\begin{abstract}
Abbreviations: ALT, alanine aminotransferase; aPTT, activated partial thromboplastin time; AST, aspartate aminotransferase; AUCs, areas under the curve; ED, emergency department; Hb, hemoglobin; hcDNA, histone-complexed DNA; INR, international normalized ratio; ISS, Injury Severity Score; MODS, multi-organ dysfunction syndrome; NLR, neutrophil-to-lymphocyte ratio; PLR, platelet-to-lymphocyte ratio; PT, pediatric trauma; ROC, receiver operating characteristic; SIRS, systemic inflammatory response syndrome; WBC, white blood cell.
\end{abstract}

Citation: Tekin Y. Are Neutrophil-to-Lymphocyte and Platelet-to-Lymphocyte Ratios Associated with Mortality in Pediatric Trauma Patients? A Retrospective Study. Rambam Maimonides Med J 2019;10 (4):eoo22. doi:10.5041/RMMJ.10376

Copyright: (C) 2019 Yusuf Kenan Tekin. This is an open-access article. All its content, except where otherwise noted, is distributed under the terms of the Creative Commons Attribution License (http://creativecommons.org/licenses/by/3.0), which permits unrestricted use, distribution, and reproduction in any medium, provided the original work is properly cited.

Conflict of interest: No potential conflict of interest relevant to this article was reported.

*E-mail: yktekin@hotmail.com 
Impact of the Neutrophil-to-Lymphocyte Ratio and Platelet Lymph

operating characteristic (ROC) curve analysis was used to define the optimum cut-off values of the NLR and the PLR parameters for survival.

Results: The NLR, and PLR values were significantly higher in survivors than in non-survivors (NLR, $6.2 \pm 5.7$ versus $2.6 \pm 2.5, P<0.001 ; \mathrm{PLR}, 145.3 \pm 85.0$ versus $46.2 \pm 25.2, \mathrm{P}<0.001$ ). The NLR (odds ratio [OR], 3.21; $P=0.048$ ), PLR (OR, 0.90; $P=0.032)$, blood glucose (OR, 1.02; $P=0.024$ ), and Injury Severity Score (ISS) (OR, 1.28; $P=0.011$ ) were independent predictors of the mortality risk in PT patients. The area under the curve in the ROC curve analysis was 0.764 with a cut-off of 2.77 (sensitivity $70 \%$, specificity $77 \%$ ) for the NLR; and 0.928 with a cut-off of 61.83 (sensitivity $90 \%$, specificity $85 \%$ ) for the PLR.

Conclusion: Acquiring the NLR and PLR at the time of admission could be a useful predictor for mortality in PT patients.

KEY WORDS: Neutrophil-to-lymphocyte ratio, pediatric patient, platelet-to-lymphocyte ratio, trauma

\section{INTRODUCTION}

Trauma-related injury as a potential cause of death affects millions of people worldwide, especially in less developed countries. ${ }^{1}$ Moreover, it is the leading cause of mortality in pediatric trauma patients. ${ }^{2,3}$ Severe trauma-related injuries may also promote a hyper-inflammatory state (usually characterized by the presence of systemic inflammatory response syndrome [SIRS]) leading to multi-organ dysfunction syndrome (MODS), which is associated with increased mortality. 4,5

Systemic inflammatory response syndrome is usually found in multi-trauma patients on at least one day during the first 72 h.5 Although it is clear that SIRS is frequently observed after severe trauma and is related to adverse health outcomes in the adult population, the effects of early SIRS and changes in the endothelial physiology in pediatric trauma (PT) patients are not fully understood. ${ }^{4}$ There are restricted data on the proposed mechanisms of trauma-induced SIRS in the pediatric population. Unfortunately, the current understanding of the possible mechanisms in SIRS is largely based on data derived from the adult population. However, in addition to the physiological and anatomical differences between children and adults, there is a variance in patterns and mechanisms of injury. ${ }^{6}$

In previous research, several scoring systems and a variety of biomarkers, such as Boehme's score system and IL-6 and the histone-complexed DNA (hcDNA), were explored to stratify mortality risk in trauma patients. 7,8 These paradigms proved beneficial for medical triage, further resuscitation of patients, and better patient management. But only a few of these scoring systems have been used in practice routinely, partly because of relatively low clinical practicality.

The most common tests, white blood cell (WBC) count and whole blood assay, have long been used to assess SIRS in clinical practice. 9 To this day, the neutrophil-to-lymphocyte ratio (NLR) and the platelet-to-lymphocyte ratio (PLR) (as a new marker of inflammatory status) have been investigated to examine the prediction ability of systemic inflammatory response in many other disorders, including malignant cancers and pulmonary diseases. ${ }^{10-12}$ Nevertheless, very limited data have been presented on the prognostic capacity of both NLR and PLR for SIRS in PT patients.

\section{MATERIAL AND METHODS}

This retrospective study included a total of 358 PT patients admitted to the emergency department (ED) of Cumhuriyet University Hospital, between January 2010 and June 2018, due to acute trauma.

The inclusion criteria were: patients (1) with acute trauma, and (2) under the age of 19 years at the time of ED admission. Exclusion criteria were as follows: (1) patients aged 19 years and older; (2) inaccessible or missing data from the hospital system; (3) patients admitted more than 24 hours after the occurrence of trauma; (4) patients presenting with any of the following co-morbid or chronic conditions: liver disease, renal failure, uncured malignant disease of any origin, immunodeficiency, pregnancy, coagulation disorders, and/or diagnosis of SIRS or infectious disease at the time of admission.

The present study was reviewed and approved by the Ethics Committee of Cumhuriyet University 
(protocol number 2018-11/o4) and conducted in accordance with the principles of the Declaration of Helsinki. Informed consent was received from the patient and/or the patient's legal guardian.

Demographic and medical data such as the Injury Severity Score (ISS) or injury type were calculated or obtained at the time of admission.

Data for hemoglobin ( $\mathrm{Hb})$, neutrophil, monocyte, white blood cell (WBC), lymphocyte, blood glucose, alanine aminotransferase (ALT), aspartate aminotransferase (AST), activated partial thromboplastin time (aPTT), and international normalized ratio (INR) were retrospectively derived from the medical record database of the first electronic application of biochemical results.

Both the NLR and PLR were calculated at the time of admission by dividing the blood neutrophil count and the blood platelet count, respectively, by the lymphocyte count. The data regarding absolute neutrophil count and absolute lymphocyte count were obtained from the first routine blood assay at the time of admission.

\section{Statistical Analysis}

Data were analyzed with the SPSS software version 22.0 for Windows (IBM Corporation, New York, NY, USA). Continuous variables were presented as mean \pm standard deviation (SD) as appropriate, and categorical variables as numbers (percentages). The Mann-Whitney $U$ test was used for continuous variables, and categorical variables were compared with the Fisher exact test, to assess the significance of intergroup differences.

After performing a stepwise logistic regression analysis to determine the predictive factors for the mortality risk of post-traumatic SIRS, a receiver operating characteristic (ROC) curve analysis and associated areas under the curve (AUCs) with 95\% CIs were separately plotted to define the optimum cut-off point both for the NLR and the PLR. A $P$ value $<0.05$ was considered statistically significant.

\section{RESULTS}

A total of 358 PT patients (Table 1) were recruited in this study. Nine patients (2.5\%) died within 24 hours without undergoing surgical intervention. Seventy-eight (21.8\%) patients underwent surgery, with the most frequent operations being to the extremities, cranium, chest, and/or abdomen (data not shown). Four of the patients $(4 / 78,5.1 \%)$ died between 24 hours and 7 days following surgery.

Table 1 shows that there was no significant difference between the two groups of survivors and non-survivors in terms of gender, age, and traffic accident as injury mechanism (male, 226/4, female, $119 / 9$; mean age [SD], 8.98 [5.52] versus 8.62 [5.38]; and 280/13, respectively, $P>0.05$ ).

Referring to Table 2, the Hb, monocyte, NLR, and PLR levels were found to be statistically higher in survivors than in non-survivors (Hb, mean [SD] $12.9[1.7] \mathrm{g} / \mathrm{dL}$ versus 10.7 [2.3] g/dL, $P<0.001$;

Table 1. Demographic Characteristics of the Survivors, Non-survivors, and Total Sample.

\begin{tabular}{|c|c|c|c|c|}
\hline Characteristics & $\begin{array}{l}\text { Total Sample } \\
\qquad(n=358)\end{array}$ & $\begin{array}{c}\text { Survivors } \\
(n=345)\end{array}$ & $\begin{array}{c}\text { Non-survivors } \\
(n=13)\end{array}$ & $\begin{array}{c}P \text { Value } \\
\text { (Fisher's } \\
\text { exact test) }\end{array}$ \\
\hline Age, year $($ mean $\pm S D)$ & $8.96 \pm 5.51$ & $8.98 \pm 5.52$ & $8.62 \pm 5.38$ & $0.798^{*}$ \\
\hline \multicolumn{5}{|l|}{ Gender, $n$ (\%) } \\
\hline Male & $235(65.6)$ & 226 & $4(30.8)$ & 0.100 \\
\hline Female & $123(34.4)$ & $119(65.5)$ & $9(69.2)$ & \\
\hline \multicolumn{5}{|c|}{ Mechanisms of injuries, $n(\%)$} \\
\hline Traffic accident & $293(81.8)$ & $280(81.2)$ & $13(100.0)$ & 0.137 \\
\hline Others $†$ & $65(28.2)$ & $65(18.8)$ & $0(00.0)$ & \\
\hline
\end{tabular}


Table 2. Mean Values of Blood Work for Survivors versus Non-survivors.

\begin{tabular}{|lcc|c|}
\hline Variables & $\begin{array}{c}\text { Survivors } \\
(n=345, \text { mean } \pm S D)\end{array}$ & $\begin{array}{c}\text { Non-survivors } \\
(n=13, \text { mean } \pm S D)\end{array}$ & $\begin{array}{c}P \text { value } \\
(\text { Mann-Whitney } U \text { test })\end{array}$ \\
\hline White blood cell $\left(10^{3} / \mu \mathrm{L}\right)$ & $15.3 \pm 5.7$ & $25.0 \pm 13.7$ & 0.026 \\
Hemoglobin $(\mathrm{g} / \mathrm{dL})$ & $12.9 \pm 1.7$ & $10.7 \pm 2.3$ & $<0.001$ \\
Platelet $\left(10^{3} / \mu \mathrm{L}\right)$ & $315.7 \pm 100.2$ & $301.0 \pm 107.7$ & 0.606 \\
Neutrophil $\left(10^{3} / \mu \mathrm{L}\right)$ & $11.8 \pm 5.3$ & $15.0 \pm 6.9$ & 0.007 \\
Lymphocyte $\left(10^{3} / \mu \mathrm{L}\right)$ & $3.0 \pm 2.0$ & $9.3 \pm 8.7$ & 0.001 \\
Monocyte $\left(10^{3} / \mu \mathrm{L}\right)$ & $1.2 \pm 6.5$ & $1.0 \pm 0.9$ & $<0.001$ \\
Neutrophil-to-lymphocyte ratio & $6.2 \pm 5.7$ & $2.6 \pm 2.5$ & $<0.001$ \\
Platelet-to-lymphocyte ratio & $145.3 \pm 85.0$ & $46.2 \pm 25.2$ & $<0.001$ \\
Lymphocyte-to-monocyte ratio & $4.2 \pm 2.9$ & $26.5 \pm 56.2$ & 0.177 \\
Mean platelet volume-to-platelet count & $0.03 \pm 0.02$ & $0.04 \pm 0.02$ & 0.421 \\
Blood glucose (mg/dL) & $124.7 \pm 37.3$ & $289.9 \pm 145.6$ & 0.002 \\
ALT (IU/L) & $54.5 \pm 110.2$ & $273.2 \pm 309.7$ & 0.026 \\
AST (IU/L) & $100.0 \pm 196.5$ & $507.3 \pm 577.6$ & 0.026 \\
aPTT (seconds) & $28.8 \pm 4.4$ & $37.9 \pm 12.4$ & 0.020 \\
INR & $1.1 \pm 0.1$ & $1.5 \pm 0.4$ & 0.004 \\
ISS & $11.7 \pm 7.4$ & $37.0 \pm 12.7$ & $<0.001$ \\
\hline ALT, alanine aminotransferase; aPTT & & \\
\hline
\end{tabular}

ALT, alanine aminotransferase; aPTT, activated partial thromboplastin time; AST, aspartate aminotransferase; INR, International normalized ratio; ISS, Injury Severity Score.

monocyte, $1.2[6.5] 10^{3} / \mu \mathrm{L}$ versus 1.0 [0.9] $10^{3} / \mu \mathrm{L}$, $P<0.001$; NLR, 6.2 [5.7] versus 2.6 [2.5], $P<0.001$; PLR, 145.3 [85] versus 46.2[25.2] $P<0.001$, respectively). Furthermore, mean values of the WBC, neutrophil, lymphocyte, blood glucose, ALT, AST, aPTT, INR levels and ISS scores were higher in nonsurvivors than in survivors (WBC, mean [SD] 15.3 [5.7] $10^{3} / \mu \mathrm{L}$ versus 25.0 [13.7] 103/ $\mu \mathrm{L}, P=0.026$; neutrophil, 11.8 [5.3] 103/ $\mu \mathrm{L}$ versus 15.0 [6.9] $10^{3} / \mu \mathrm{L}, P=0.007$; lymphocyte, 3.0 [2.0] $10^{3} / \mu \mathrm{L}$ versus 9.3 [8.7] 103/ $\mu \mathrm{L}, P=0.001$; blood glucose, $124.7[37.3] \mathrm{mg} / \mathrm{dL}$ versus 289.9 [145.6] $\mathrm{mg} / \mathrm{dL}$, $P=0.002 ;$ ALT, $54.5[110.2] \mathrm{IU} / \mathrm{L}$ versus 273.2 [309.7] IU/L, $P=0.026 ;$ AST, 100.0 [196.5] IU/L versus $507.3[577.6] \mathrm{IU} / \mathrm{L}, P=0.026$; aPTT, 28.8 [4.4] s versus 37.9 [12.4] s, $P=0.020 ;$ INR, 1.1 [0.1] versus 1.5 [0.4], $P=0.004$; and ISS, 11.7 [7.4] versus 37.0 [12.7], $P<0.001$, respectively). However, there was no significant difference between survivors and non-survivors in terms of lymphocyte-to-monocyte ratio and mean platelet volume-to-platelet count values.
Logistic regression analysis showed that the NLR (odds ratio [OR], 3.21; $P=0.048$ ), PLR (OR, 0.90; $P=0.032)$, blood glucose (OR, 1.02; $P=0.024)$, and ISS (OR, $1.28 ; P=0.011)$ were independently predictive of mortality risk in PT patients (Table 3 ).

The ROC curve analysis included the NLR and PLR, which were significantly associated with mortality in bivariate analyses. Referring to Table 4 and Figure 1, the NLR had an area of 0.764 , a cut-off of 2.77 , a sensitivity of $70 \%$, and a specificity of $77 \%$; the PLR had an area of 0.928 , a cut-off of 61.83 , a sensitivity of $90 \%$, and a specificity of $85 \%$.

\section{DISCUSSION}

This study showed that PT patients with early fatal outcomes had higher blood sugar, and lower PLR and NLR levels on admission, indicating that these parameters may be early predictors of mortality. Moreover, the cut-off values of NLR and PLR have been proposed in this study to estimate the mortality risk in PT patients. Based on a literature review, this 
Table 3. Variables Related with Mortality in Logistic Regression Analysis $(n=358)$.

\begin{tabular}{|lcc|c|}
\hline Independent Variables & Odds Ratio & 95\% Confidence Interval & $\boldsymbol{P}$ Value \\
\hline Neutrophil-to-lymphocyte ratio & 3.21 & $1.01-10.22$ & 0.048 \\
Platelet-to-lymphocyte ratio & 0.90 & $0.81-0.99$ & 0.032 \\
Lymphocyte-to-monocyte ratio & 2.07 & $0.97-4.39$ & 0.059 \\
Blood glucose (mg/dL) & 1.02 & $1.00-1.05$ & 0.024 \\
ISS & 1.28 & $1.06-1.54$ & 0.011 \\
\hline
\end{tabular}

ISS, Injury Severity Score.

Table 4. Cut-off Value, Sensitivity, and Specificity of Neutrophil-to-Lymphocyte Ratio and Platelet-toLymphocyte Ratio for Predicting of Mortality in Prognosis of Pediatric Trauma.

\begin{tabular}{|l|c|c|}
\hline Parameter & $\begin{array}{c}\text { Neutrophil-to-Lymphocyte } \\
\text { Ratio (NLR) }\end{array}$ & $\begin{array}{c}\text { Platelet-to-Lymphocyte Ratio } \\
\text { (PLR) }\end{array}$ \\
\hline Cut-off value & 2.77 & 61.83 \\
Sensitivity & 0.70 & 0.90 \\
Specificity & 0.77 & 0.85 \\
AUC (95\% Cl) & $0.764(0.636-0.891)$ & $0.928(0.871-0.985)$ \\
\hline
\end{tabular}

AUC, area under the curve; $\mathrm{Cl}$, confidence interval.

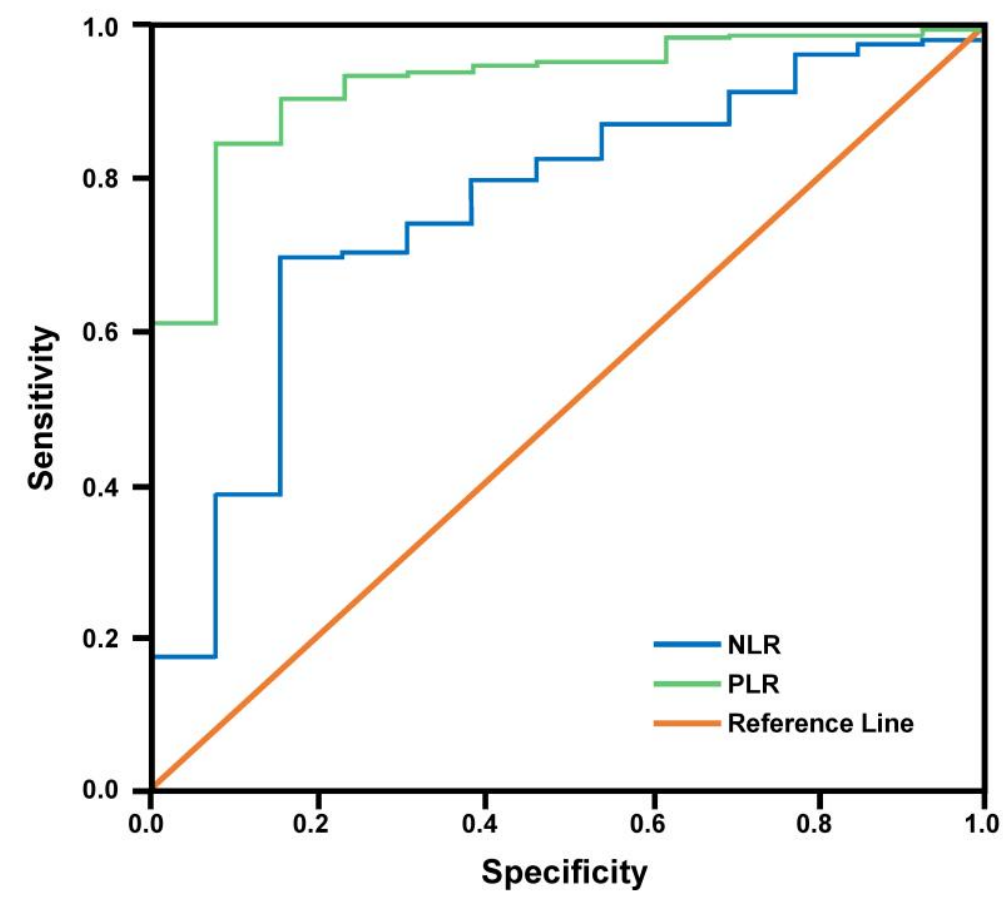

Figure 1. Receiver Operating Characteristic (ROC) Curve of Neutrophil-to-Lymphocyte Ratio (NLR) and Plateletto-Lymphocyte Ratio (PLR) for the Prediction of Prognosis of Mortality in Patients with Pediatric Trauma. 
is the first study demonstrating a significant association between a high mortality rate and NLR, PLR, and blood glucose among PT patients. As reported in many previous studies, a higher ISS was also predictive of poor outcomes in the present study. ${ }^{13,14}$

It should be noted that the cascade of hyperinflammation of SIRS may occur within 30 minutes after the initial of injury.15 Systemic inflammatory response syndrome (SIRS) is one of the most common post-traumatic injury complications and has previously been shown to be a remarkable predictor of mortality in trauma patients. ${ }^{16,17}$ Also reported previously, post-traumatic SIRS was not only associated with higher mortality of trauma patients, but also with a higher rate of nosocomial infection and longer length of hospital stay. ${ }^{17,18}$ In this regard, it is very important to determine the patients at risk for developing SIRS by identifying reliable biomarkers. In the present study, WBC count, neutrophil count, lymphocyte count, blood glucose, ALT, AST, aPTT, INR, and ISS were remarkably higher in nonsurvivors, whereas hemoglobin, monocyte count, NLR, and PLR levels were significantly higher in survivors. Logistic regression analysis showed that NLR (OR, 3.21; P=0.048), PLR (OR, 0.90; $P=0.032)$, blood glucose (OR, 1.02; $P=0.024)$, and ISS (OR, 1.28; $P=0.011)$ were independently predictive of the risk of mortality in PT patients.

Additionally, this study showed that blood glucose levels were an independent factor for the prognosis of PT patient mortality. This finding was consistent with previous reports indicating that hyperglycemia caused by traumatic brain injury or multiple trauma was associated with poor outcomes in the pediatric population..$^{19-21}$

In response to severe tissue trauma such as acute lung injury, neutrophils-as part of a systemic inflammatory response-not only rapidly contribute to inflammation activation, but also may cause organ failure. ${ }^{22}$ Platelets, as cellular effectors of both inflammation and thrombosis, are also activated after traumatic injuries. ${ }^{23}$ As reported in previous studies, the platelet-neutrophil interaction may contribute to neutrophil recruitment, which leads to tissue inflammation. ${ }^{24}$ In addition to contributing to the inflammatory response, a decrease in total lymphocyte count has been found to be related to MODS in adult patients with a traumatic injury. ${ }^{23,25}$ However, in the present study, trauma-induced lymphocytosis was observed in the first blood sam- ples taken from PT patients. One possible explanation for this is that a neutrophilic response is expected in some cases of acute and stress-induced inflammatory conditions. However, some stressful and urgent medical conditions, such as non-surgical trauma, cardiac emergencies, sickle cell anemia crises, abdominal pain, and obstetric emergencies, may lead to a transient type of lymphocytosis instead of the neutrophilic response. ${ }^{26,27}$

Recently, and similar to this study's findings, it was reported that, except for patients with posttraumatic SIRS, lymphocytosis and elevated WBC count among patients with traumatic injury were significantly associated with ISS and a higher mortality rate. ${ }^{28}$ Moreover, as demonstrated in some other studies, NLR is closely linked to mortality among patients with severe traumatic injury or critically traumatic brain injury. 29,30

While reduced lymphocyte count is related to immunosuppression, increased platelet and neutrophil counts reflect systemic inflammation. Therefore, NLR and PLR, which combine both immunosuppression and inflammation, might be more reliable biomarkers than WBC or platelet counts.

This study had certain limitations. It was retrospective and included a small number of PT patients; hence the mortality ratio was also relatively low. In addition, laboratory findings for certain clinical and inflammatory markers, such as interleukin6 , TNF- $\alpha$, etc., were not available. Therefore, the findings of this current study should be validated in further prospective studies. For this purpose, we have a project to verify the results of the study.

\section{CONCLUSION}

In conclusion, the NLR and PLR at the time of admission could be useful predictors for mortality in PT patients. This study indicates that NLR and PLR, as well as blood glucose and ISS at the time of admission, were independently associated with post-traumatic SIRS. Further studies should be carried out to validate the role of those parameters to optimize treatment strategy in PT patients.

\section{REFERENCES}

1. Vecino-Ortiz AI, Jafri A, Hyder AA. Effective interventions for unintentional injuries: a systematic review and mortality impact assessment among the poorest billion. Lancet Glob Health 2018;6:e523-4. $\underline{\text { Crossref }}$ 
2. Christian CW, Sege RD; The Committee on Child Abuse and Neglect; The Committee on Injury, Violence, and Poison Prevention; The Council on Community Pediatrics. American Academy of Pediatrics. Policy statement--child fatality review. Pediatrics 2010;126:592-6. $\underline{\text { Crossref }}$

3. Centers for Disease Control and Prevension (CDC). Vital signs: unintentional injury deaths among persons aged 0-19 years - United States, 2000-2009. MMWR Morb Mortal Wkly Rep 2012;61:2706.

4. Brohi K, Cohen MJ, Ganter MT, et al. Acute coagulopathy of trauma: hypoperfusion induces systemic anticoagulation and hyperfibrinolysis. J Trauma 2008;64:1211-17. Crossref

5. Butcher N, Balogh ZJ. The definition of polytrauma: the need for international consensus. Injury 2009; 40(Suppl 4):S12-22. Crossref

6. Whittaker B, Christiaans SC, Altice JL, et al. Early coagulopathy is an independent predictor of mortality in children after severe trauma. Shock 2013;39:4216. $\underline{\text { Crossref }}$

7. Sapan HB, Paturusi I, Jusuf I, et al. Pattern of cytokine (IL-6 and IL-10) level as inflammation and antiinflammation mediator of multiple organ dysfunction syndrome (MODS) in polytrauma. Int $\mathrm{J}$ Burns Trauma 2016;6:37-43.

8. Russell RT, Christiaans SC, Nice TR, et al. Histonecomplexed DNA fragments levels are associated with coagulopathy, endothelial cell damage, and increased mortality after severe pediatric trauma. Shock 2018; 49:44-52. Crossref

9. Chang DC, Cornwell EE 3rd, Phillips J, Paradise J, Campbell K. Early leukocytosis in trauma patients: what difference does it make? Curr Surg 2003;60: 632-5. Crossref

10. Yao Y, Yuan D, Liu H, Gu X, Song Y. Pretreatment neutrophil to lymphocyte ratio is associated with response to therapy and prognosis of advanced nonsmall cell lung cancer patients treated with first-line platinum-based chemotherapy. Cancer Immunol Immunother 2013;62:471-9. Crossref

11. Wang SC, Chou JF, Strong VE, Brennan MF, Capanu M, Coit DG. Pretreatment neutrophil to lymphocyte ratio independently predicts disease-specific survival in resectable gastroesophageal junction and gastric adenocarcinoma. Ann Surg 2016;263:292-7. Crossref

12. Yang W, Wang $\mathrm{X}$, Zhang $\mathrm{W}$, et al. Neutrophillymphocyte ratio and platelet-lymphocyte ratio are 2 new inflammatory markers associated with pulmonary involvement and disease activity in patients with dermatomyositis. Clin Chim Acta 2017;465:11-16. $\underline{\text { Crossref }}$
13. Baker SP, O’Neill B, Haddon W Jr, Long WB. The injury severity score: a method for describing patients with multiple injuries and evaluating emergency care. J Trauma 1974;14:187-96.

14. Brown JB, Gestring ML, Leeper CM, et al. The value of the Injury Severity Score in pediatric trauma: time for a new definition of severe injury? J Trauma Acute Care Surg 2017;82:995-1001. Crossref

15. Lord JM, Midwinter MJ, Chen YF, et al. The systemic immune response to trauma: an overview of pathophysiology and treatment. Lancet 2014;384:1455-65. Crossref

16. Napolitano LM, Ferrer T, McCarter RJ Jr, Scalea TM. Systemic inflammatory response syndrome score at admission independently predicts mortality and length of stay in trauma patients. J Trauma 2000;49: 647-52. $\underline{\text { Crossref }}$

17. Jacome T, Tatum D. Systemic inflammatory response syndrome (SIRS) score independently predicts poor outcome in isolated traumatic brain injury. Neurocrit Care 2018;28:110-16. Crossref

18. Easton R, Balogh ZJ. Peri-operative changes in serum immune markers after trauma: a systematic review. Injury 2014;45:934-41. Crossref

19. Sharma D, Jelacic J, Chennuri R, Chaiwat O, Chandler W, Vavilala MS. Incidence and risk factors for perioperative hyperglycemia in children with traumatic brain injury. Anesth Analg 2009;108:81-9. Crossref

20. Chong SL, Harjanto S, Testoni D, et al. Early hyperglycemia in pediatric traumatic brain injury predicts for mortality, prolonged duration of mechanical ventilation, and intensive care stay. Int $\mathrm{J}$ Endocrinol 2015;2015:719476. $\underline{\text { Crossref }}$

21. Elkon B, Cambrin JR, Hirshberg E, Bratton SL. Hyperglycemia: an independent risk factor for poor outcome in children with traumatic brain injury. Pediatr Crit Care Med 2014;15:623-31. $\underline{\text { Crossref }}$

22. Xu J, Guardado J, Hoffman R, et al. IL33-mediated ILC2 activation and neutrophil IL5 production in the lung response after severe trauma: a reverse translation study from a human cohort to a mouse trauma model. PLoS Med 2017;14:e1002365. Crossref

23. Morris RS, Schaffer BS, Lundy JB, et al. Immunopathological response to severe injury: platelet activation and the Th-17 immune response. Blood Coagul Fibrinolysis 2018;29:48-54. $\underline{\text { Crossref }}$

24. Soehnlein O. Decision shaping neutrophil-platelet interplay in inflammation: from physiology to intervention. Eur J Clin Invest 2018;48:e12871. Crossref

25. Manson J, Cole E, De'Ath HD, et al. Early changes within the lymphocyte population are associated with 
the development of multiple organ dysfunction syndrome in trauma patients. Crit Care 2016;20:176. Crossref

26. Thommasen HV, Boyko WJ, Montaner JSG, Russell JA, Johnson DR, Hogg JC. Absolute lymphocytosis associated with nonsurgical trauma. Am J Clin Pathol 1986;86:480-3. Crossref

27. Karandikar NJ, Hotchkiss EC, McKenna RW, Kroft SH. Transient stress lymphocytosis: an immunophenotypic characterization of the most common cause of newly identified adult lymphocytosis in a tertiary hospital. Am J Clin Pathol 2002;117:819-25. Crossref
28. Pinkerton PH, McLellan BA, Quantz MC, Robinson JB. Acute lymphocytosis after trauma--early recognition of the high-risk patient? J Trauma 1989;29:74951. $\underline{\text { Crossref }}$

29. Dilektasli E, Inaba K, Haltmeier T, et al. The prognostic value of neutrophil-to-lymphocyte ratio on mortality in critically ill trauma patients. J Trauma Acute Care Surg 2016;81:882-8. Crossref

30. Chen W, Yang J, Li B, et al. Neutrophil to lymphocyte ratio as a novel predictor of outcome in patients with severe traumatic brain injury. J Head Trauma Rehabil 2018;33:E53-9. $\underline{\text { Crossref }}$ 